\title{
Investigation on the Influence of Ultra-violet Rays on the Physiological Activities of Azotobacter. II.
}

On the Stimulation of Azotobacter Chroococcum by Ultra-violet Rays.

By

Arao Itano and Akira Matuura.

(Received June 19, 1934.)

The preceding paper (Jour. Agr. Chem. Soc., Japan. X: 477 485, 1934.) dealt with the lethal action of ultra-violet rays on Azotobacter chroococcum, and in the course of investigation, it was found that a short exposure stimulated the physiological activity of Azotobacter, and in this investigation, the following points were investigated and the results are reported: 1. Rate of stimulation by different length of exposure, determined by the number of cells and also by the change of $\mathrm{pH}$ in the medium ; 2 . Mechanism of stimulation, the influence of ultra-violet rays on the physical properties of medium and their subsequent influence on Azotobacter; 3. Manner of exposure, continuous or intermittent.

The method used is the same as described previously, using Hanovia mercury lamp as the source of ultra-violet rays and exposed Azotobacter chroococcum for different intervals in Ashby's solution medium placed in Erlenmyer flask of hard glass, and obtained the following results : 1 . The greatest number of cells was obtained by one minute exposure, and the longer exposure caused the decrease of cells. The change of $\mathrm{pH}$ in medium was greatest on one minute exposure and the reaction became acid; 2 . It was ascertained that the increase of cells was brought about chiefly by the action of ultra-violet rays such other factors as the heat rays, visible rays and the change of physical properties of medium have little influence; 3 . The influence of ultra-violet rays on the physical properties of medium such as electrical conductance, $\mathrm{pH}$, osmotic pressure, viscosity and surface tension, was determined and found that the change of electrical conductance, $\mathrm{pH}$ and osmotic pressure was comparatively greater than that of other properties, and the reducing action was noted, although it is not sufficient to consider the reduction as an important factor which brings about the stimulation; 4. So far as the manner of exposure is concerned, the better stimulation was obtained by the continuous exposure than by the intermittent within one minute, but when the exposure was longer, the intermittent exposure was better owing to the lethal action of continuous exposure. 